\title{
MATEMÁTICA DE CRÉDITO AO CONSUMIDOR
}

\section{EdMundo Éboli Bonini *}

Desde que se iniciou no Brasil a chamada "reforma do mercado de capitais" êste setor passou a concentrar as atenções de todos os segmentos da economia nacional. As diferentes entidades que compõem o setor financeiro começaram a ser alvo de especial curiosidade. As Bôlsas de Valôres dos Bancos Comerciais, dos Bancos de Investimentos e sobretudo das Financeiras começaram a ser objeto de atenção especial.

Essa atenção não se circunscreveu às suas funções como peças de um mecanismo maior. Ao contrário, a mecânica de funcionamento de cada uma delas, sobretudo daquelas que foram particularmente influenciadas pela mencionada reforma, vem despertando inusitado interêsse. $O$ funcionamento de entidades como a Bôlsa, as Financeiras ou os Bancos de Investimento, a par das sutilezas próprias de qualquer emprêsa, pressupõe também tôda uma técnica de análise, sem a qual não seria êle satisfatório.

Nessa matéria tem papel destacado a matemática financeira, indispensável para a correta tomada de decisões

* Professor Contratado do Departamento de Métodos Quantitativos da Escola de Administração de Emprêsas de São Paulo, da Fundação Getúlio Vargas. Professor das Faculdades de Ciências Econômicas e Administrativas da Universidade de São Paulo, Faculdade de Ciências Econômicas de São Paulo, Faculdade de Ciências Econômicas de São Luís, Escola de Engenharia Mauá e Faculdade de Ciências Econômicas da Universidade Mackenzie. 
sôbre empréstimos ou inversões efetuadas por essas emprêsas.

O presente artigo aborda a questão do crédito ao consumidor e a interveniência das financeiras em operações dêsse tipo.

Em função da carência de capital para a aquisição de produtos de consumo durável e veículos, as financeiras podem emitir títulos (letras de câmbio), para a obtenção de capital e entregá-las aos consumidores de capital para a aquisição de bens.

O fluxograma apresenta-se da seguinte forma:

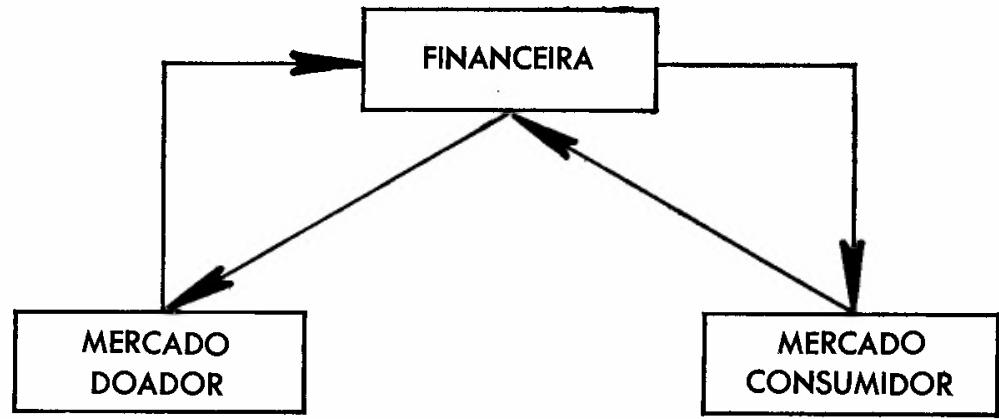

Portanto, temos duas fases do processo do fluxo do capital:

1. Obtenção por parte da financeira do capital no mercado.

2. Entrega do capital ao mercado consumidor (usuários).

EXEMPLOS DE CÁLCULOS

A financeira para a obtenção de dinheiro no mercado doador, deverá, evidentemente, pagar algumas comissões, taxas e também apresentar uma certa rentabilidade, tais como:

- Rentabilidade para o mercado doador (Letra de Câmbio).

- Corretagem para o vendedor da Letra de Câmbio.

- Corretagem de Aceite (Receita da financeira). 
- Outras Corretagens (Receitas das financeiras).

- Impôsto sôbre Operações financeiras (IOF).

Iremos, a título de exemplo, considerar as seguintes taxas:

- $32 \%$ ao ano

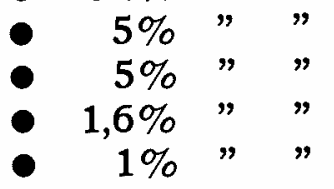

Consideremos que o Mercado Consumidor deva . . . . . . $\mathrm{NCr} \$ 12.000,00$, a serem pagos à financeira em 12 promissórias de $\mathrm{NCr} \$ 1.000,00$ cada. Para a obtenção dêste capital, a financeira lançará no mercado doador letras de câmbio. Suponhamos que sejam 12 letras de câmbio de $\mathrm{NCr} \$ 1.000,00$. Como o prazo mínimo de vencimento da letra é de 6 meses, teremos que a primeira terá o valor de $\mathrm{NCr} \$ 6.000,00$ e as sucessivas de $\mathrm{NCr} \$ 1.000,00$, de acôrdo com o seguinte diagrama:

\begin{tabular}{cccccccc} 
& 6 meses & 7 & 8 & 9 & 10 & 11 & 12 \\
\hline & 1 & 1 & 1 & 1 & 1 & 1 & 1 \\
\hline Hoje & 6.000 & 1.000 & 1.000 & 1.000 & 1.000 & 1.000 & 1.000
\end{tabular}

1. A que valor serão colocadas no Mercado Doador as letras de câmbio? De acôrdo com o primeiro exemplo, temos uma taxa $\alpha=32 \%$ a.a. Assim, a letra de câmbio com o vencimento para 6 meses terá o valor no mercado doador de:

$$
\begin{aligned}
& \mathrm{K}_{1}+\frac{\alpha}{2} \mathrm{~K}_{1}=1=>\mathrm{K}_{1}\left(1+\frac{\alpha}{2}\right)=1=>\mathrm{K}_{1}=\frac{1}{1+\frac{\alpha}{2}} \\
& \mathrm{~K}_{1}=\frac{1}{1+\frac{0,32}{2}}=\frac{1}{1+0,16}=\frac{1}{1,16}=0,862069
\end{aligned}
$$

Sendo que $K_{1}$ é o coeficiente que deverá multiplicar as letras de câmbio com o vencimento para 6 meses. Portanto, teremos:

$$
6.000,00 \times 0,862069=5.172,414(\text { Anexo 1) }
$$


Para as outras letras com vencimentos sucessivos, os coeficientes serão:

$$
\begin{aligned}
& \mathrm{K}_{2}+\frac{\alpha}{2} \mathrm{~K}_{2}+\frac{\alpha}{12} \mathrm{~K}_{2}=1 \\
& \mathrm{~K}_{2}=\frac{1}{1+\frac{\alpha}{2}+\frac{\alpha}{12} \cdot 1}=\frac{1}{1+\frac{0,32}{2}+\frac{0,32}{12} \cdot 1}=0,842694
\end{aligned}
$$

e assim, genèricamente, teremos:

$$
\mathrm{K}_{\mathrm{j}+1}=\frac{1}{1+\frac{\alpha}{2}+\frac{\alpha}{12} \cdot \mathrm{j}}
$$

$$
j=[0,1,2, \ldots \ldots, 18]
$$

e os respectivos valôres serão:

$$
\begin{aligned}
\mathrm{K}_{3}=\frac{1}{1,21334} & =0,824171 \\
\mathbf{K}_{4} & =0,806445 \\
\mathbf{K}_{5} & =0,789465 \\
\mathrm{~K}_{6} & =0,773186 \\
\mathbf{K}_{7} & =0,757564 \\
\mathbf{K}_{8} & =0,742561 \\
\mathbf{K}_{9} & =0,728141 \\
\mathbf{K}_{10} & =0,714270 \\
\mathbf{K}_{11} & =0,700918 \\
\mathbf{K}_{12} & =0,688056 \\
\mathbf{K}_{13} & =0,675657 \\
\mathbf{K}_{14} & =0,663698 \\
\mathbf{K}_{15} & =0,652154 \\
\mathbf{K}_{16} & =0,641005 \\
\mathbf{K}_{17} & =0,630230 \\
\mathbf{K}_{18} & =0,619813 \\
\mathbf{K}_{19} & =0,609734
\end{aligned}
$$


Temos, então, no Anexo 1, o dinheiro proveniente do "mercado doador".

2. A financeira terá de pagar uma taxa de corretagem $(\beta)$ sôbre a venda das letras de câmbio. Consideremos que a taxa seja da ordem de $5 \%$ ao ano. Assim, temos que para a primeira letra a financeira pagará 0,025. Observar que as comissões são pagas sôbre o líquido das letras. Os outros coeficientes para a obtenção da corretagem serão:

$$
\begin{aligned}
& \lambda_{1}=\frac{0,05}{2}+\frac{0,05}{12} \cdot 0=0,025 \\
& \lambda_{2}=\frac{0,05}{2}+\frac{0,05}{12} \cdot 1=0,025+0,004166 \cdot 1=0,029166
\end{aligned}
$$

e assim, genèricamente, teremos:

$$
\begin{array}{ccc}
\lambda_{j+1}=\frac{\beta}{2}+\frac{\beta}{12} \cdot j & j=[0,1,2, \ldots \ldots, 18] \\
\lambda_{3}=0,033332 & \lambda_{9}=0,058328 & \lambda_{15}=0,083324 \\
\lambda_{4}=0,037498 & \lambda_{10}=0,062494 & \lambda_{16}=0,087490 \\
\lambda_{5}=0,041664 & \lambda_{11}=0,066660 & \lambda_{17}=0,091656 \\
\lambda_{6}=0,045830 & \lambda_{12}=0,070826 & \lambda_{18}=0,095822 \\
\lambda_{7}=0,049996 & \lambda_{13}=0,074992 & \lambda_{19}=0,099988 \\
\lambda_{8}=9,054162 & \lambda_{14}=0,079158 &
\end{array}
$$

Temos no Anexo 2 a corretagem a ser paga pela financeira, sendo que o mesmo foi obtido multiplicando-se cada parcela de capital recebido do mercado doador pela correspondente taxa do prazo $(\lambda)$. Assim, temos que, por exemplo, para o dinheiro proveniente do mercado pelas 6 letras de valor nominal NCr $\$ 1.000,00$ cada uma e que a Financeira recebeu NCr\$5.172,414 deverá pagar ao corretor $\left(\mu \lambda_{1}\right)$ ou seja NCr\$ 5.172,414 $\times 0,025=129,310350$; a segunda letra com vencimento para 7 meses e de valor nominal de $\mathrm{NCr} \$ 1.000,00$ a financeira receberá do mercado doador $\mathrm{NCr} \$ 842,694$, conforme Anexo 1 e 
deverá pagar ao corretor $\left(\mu_{1} \lambda_{2}\right)$ ou seja $\operatorname{NCr} \$ 842,694 \times$ $\times 0,029166=24,578013$, conforme Anexo 2 e assim por diante.

3. A financeira, para aceitar a operação de crédito, cobrará uma comissão de $5 \%$ ao ano, sendo que esta comissão comportar-se-á de acôrdo com a corretagem, uma vez que a mesma é de $5 \%$ ao ano, por hipótese.

4. A financeira cobrará também outras comissões que, somadas, serão $1,6 \%$ ou 0,016 e, desdobradas, serão: $0,016=(0,005+0,005+0,005+0,001)$. Portanto, teremos de obter os coeficientes para: $0,005(\gamma)$ e 0,001 ( $\delta)$. Assim, temos:

Para $0,005(\gamma)$

$$
\begin{aligned}
& \mathbf{M}_{\mathbf{j}+1}=\frac{\gamma}{2}+\frac{\gamma}{12} \mathbf{j} \\
& j=[0,1,2, \ldots, 18] \\
& \mathrm{M}_{1}=\frac{0,005}{2}+\frac{0,005}{12} \cdot 0=0,0025+0,0004166 \cdot 0=0,0025000 \\
& \mathbf{M}_{2}=0,0029166 \\
& \mathrm{M}_{11}=0,0066600 \\
& \mathbf{M}_{3}=0,0033320 \\
& M_{12}=0,0070826 \\
& \mathbf{M}_{4}=0,0037498 \\
& \mathrm{M}_{13}=0,0074992 \\
& \mathbf{M}_{5}=0,0041664 \\
& \mathbf{M}_{14}=0,0079158 \\
& \mathbf{M}_{6}=0,0045830 \\
& M_{15}=0,0083324 \\
& \mathbf{M}_{7}=0,0049996 \\
& M_{16}=0,0087490 \\
& \mathbf{M}_{\mathbf{8}}=0,0054162 \\
& \mathbf{M}_{17}=0,0091656 \\
& \mathrm{M}_{9}=0,0058328 \\
& M_{18}=0,0095822 \\
& M_{10}=0,0062494 \\
& \mathrm{M}_{19}=0,0099988
\end{aligned}
$$

Assim, temos o Anexo 3, obtido através da multiplicação dos coeficientes $\mathbf{M}_{\mathbf{j + 1}}$ pelos capitais líquidos provenientes do mercado doador (Anexo 1). 
Para a taxa: $0,001(\delta)$

$$
\begin{aligned}
& \mathrm{N}_{\mathrm{j}+1}=\frac{\delta}{2}+\frac{\delta}{12} \mathrm{j}, \quad[\mathrm{j}=0,1,2, \ldots ., 18] \\
& \mathrm{N}_{1}=\frac{0,001}{2}+\frac{0,001}{12} \cdot 0=0,0005+0,00008333 \cdot 0=0,00050000 \\
& \mathrm{~N}_{2}=0,00058333 \\
& \mathrm{~N}_{8}=0,00108331 \\
& \mathbf{N}_{14}=0,00158329 \\
& \mathrm{~N}_{3}=0,00066666 \\
& \mathbf{N}_{9}=0,00116664 \\
& \mathbf{N}_{15}=0,00166662 \\
& \mathbf{N}_{4}=0,00074999 \\
& \mathrm{~N}_{10}=0,00124997 \\
& \mathrm{~N}_{16}=0,00147995 \\
& \mathrm{~N}_{5}=0,00083332 \\
& \mathrm{~N}_{11}=0,00133330 \\
& \mathrm{~N}_{17}=0,00183328 \\
& \mathrm{~N}_{6}=0,00091665 \\
& \mathbf{N}_{12}=0,00141663 \\
& \mathbf{N}_{18}=0,00191551 \\
& \mathrm{~N}_{7}=0,00099998 \\
& N_{13}=0,00149996 \\
& \mathrm{~N}_{19}=0,00199940
\end{aligned}
$$

Assim, temos que o Anexo 4 é obtido através da multiplicação $\mathrm{N}_{\mathrm{j}+1}$, pelos capitais líquidos provenientes do mercado doador (Anexo 1).

5. O impôsto sôbre operações financeiras (IOF) é de $1 \%$ e incide sôbre a soma dos valôres nominais das letras e da corretagem, cujos cálculos estão apresentados no Anexo 5.

COMENTÁRIOS FINAIS

I. A financeira funciona apenas como uma intermediária entre o mercado doador e o mercado consumidor. Por isso, em nosso Anexo 6, apresentamos os seguintes elementos:

(1) Capitais.

(2) Correção monetária = capitais - capitais provenientes do mercado doador (Anexo 1), que corresponde o lucro do mercado doador (lucratividade das letras de câmbio).

(3) Corretagem.

(4) Comissão da financeira.

(5) Impôsto de Operações Financeiras (IOF).

(6) Outras comissões [3 $3 \times$ Anexo (3) + Anexo (4) $]$. 
(7) Somas dos itens (2) $+(3)+(4)+(5)+(6)=$ $=$ despesas e lucratividade da financeira.

(8) Líquido $=$ capitais (1) - despesas (7), que corresponde à quantia que será dada ao mercado consumidor (usuários).

II. Determinação da taxa de juros para o mercado consumidor, isto é, para os usuários. O montante do capital líquido recebido pelo mercado consumidor deverá ser igual ao montante das prestações. Assim, temos dois critérios:

- a capitalização simples, e

- a capitalização composta.

Determinação da taxa

- Capitalização simples

$\mathbf{M}=\mathbf{M}^{\prime}$; sendo $\mathbf{M}=$ montante do capital líquido (8), Anexo 6 e $M^{\prime}=$ montante das prestações a serem pagas pelos usuários.

$$
\begin{aligned}
& M=C(1+\text { in }) \\
& M^{\prime}=C_{1}\left[n+\frac{\text { in }(n-1)}{2}\right] \\
& \quad C(1+\text { in })=C_{1}\left[n+\frac{i n(n-1)}{2}\right] \\
& \quad i=\frac{C_{1} \cdot n-C}{C n-C_{1} n \cdot \frac{(n-1)}{2}}
\end{aligned}
$$

Considerando-se $\mathrm{n}=12, \mathrm{C}_{1}=1.000,00$ e $\mathrm{C}=9.105,46$; vem:

$$
\mathrm{i}=\frac{1.000,00 \times 12-9.105,46}{9.105,46 \times 12-1.000,00 \times \frac{12 \cdot 11}{2}}=0,0669 \text { ou } 6,69 \%
$$


ao mês. Para o cálculo de outros capitais, o procedimento é análogo.

\section{- Capitalização Composta}

$\mathbf{M}=\mathbf{M}^{\prime}$; sendo $\mathbf{M}=$ montante do capital líquido (8), anexo 6 e $\mathbf{M}^{\prime}=$ montante das prestações a serem pagas pelos usuários.

$$
\begin{aligned}
& M=C(1+i)^{n} \\
& M^{\prime}=C_{1} \cdot \frac{\left(r^{n}-1\right)}{i}=C_{1} \cdot \frac{(1+i)^{n}-1}{i} \\
& C(1+i)^{n}=C_{1} \cdot \frac{(1+i)^{n}-1}{i}=>\frac{C}{C_{1}}=\frac{(1+i)^{n}-1}{(1+i)^{n}-i}=> \\
& \quad \frac{C_{1}}{C}=\frac{(1+i)^{n} \cdot i}{(1+i)^{n}-1}=\frac{a^{-1}}{n}
\end{aligned}
$$

Considerando $\mathrm{n}=12 \mid \mathrm{C}_{1}=1.000$ e $\mathrm{C}=9.105 .46$, temos: $0,1098242=\frac{1.000,00}{9.205,46}=\frac{(1+i)^{12} \cdot i}{(1+i)^{12}-1}$, observando a tabela $a^{-1}$ para $n=12$ encontramos $i$ aproximadamente igual $\mathrm{n}$

a 0,045 ou $4,5 \%$ ao mês.

Obs.: Um processo mais refinado para o cálculo da taxa na capitalização composta pode ser feito através de um processo iterativo. (Aproximaçôes sucessivas.)

Seja:

$$
\begin{aligned}
& \mathrm{C}_{1}=\mathrm{C} \cdot \frac{\mathrm{a}^{-1}}{\mathrm{n} \mid}, \text { sendo } \frac{\mathrm{a}}{\mathrm{n} \mid}=\frac{1-(1+\mathrm{i})^{-\mathrm{n}}}{\mathrm{i}} \\
& \mathrm{C}_{1}=\frac{\mathrm{C} \cdot \mathrm{i}}{\mathrm{1}=(1+\mathrm{i})^{-\mathrm{n}}}=>\mathrm{Ci}=\left[1-(1+\mathrm{i})^{-\mathrm{n}}\right] \mathrm{C}_{1} \\
& \mathrm{i}=\frac{\mathrm{C}_{1}}{\mathrm{C}}\left[1-(1+\mathrm{i})^{-\mathrm{n}}\right] \alpha
\end{aligned}
$$


Esta fórmula vai servir-nos para determinar i, por aproximações sucessivas. A aproximação in, de ordem $h$ de $i$, determina-se pela aproximação de ordem $i_{h-1}$, pela seguinte fórmula de recorrência:

$$
i_{n}=\frac{C_{1}}{C}\left[1-\left(1+i_{h-1}\right)^{-n}\right]
$$

Como ponto de partida para a primeira aproximação io de $i$, vamos estabelecer um valor menor que $i$, mas que seja próximo de seu valor.

Para isso vamos desenvolver a potência $(1+i)^{-n} \mathrm{de}$ acôrdo com a lei binomial de NEWTON.

$$
(1+i)^{-n}=1-n \cdot i+\frac{n(n+1)}{2} \cdot i^{2}-\frac{n(n+1)(n+2)}{2 \cdot 3} i^{3}+\ldots ;
$$

êste desenvolvimento apresenta uma série convergente Substituindo na igualdade $\alpha$ o têrmo $(1+i)^{-n}$ pelo desenvolvimento acima, vem:

$$
\begin{gathered}
\mathrm{i}=\frac{\mathrm{C}_{1}}{\mathrm{C}}\left[1-1+\mathrm{ni}-\frac{\mathrm{n}(\mathrm{n}+1) \mathrm{i}^{2}}{2} \mathrm{i}^{2}+\frac{\mathrm{n}(\mathrm{n}+1)(\mathrm{n}+2)}{2 \cdot 3} \cdot \mathrm{i}^{3}-\ldots\right] \\
\mathrm{i}=\frac{\mathrm{C}_{1}}{\mathrm{C}}\left[\mathrm{ni}-\frac{\mathrm{n}(\mathrm{n}+1)}{2} \cdot \mathrm{i}^{2}+\frac{\mathrm{n}(\mathrm{n}+1)(\mathrm{n}+2)}{2 \cdot 3} \cdot \mathrm{i}^{3}-\ldots\right]
\end{gathered}
$$

dividindo-se ambos têrmos por n.i, obtemos:

$$
\frac{1}{\mathrm{n}}=\frac{\mathrm{C}_{1}}{\mathrm{C}}\left[1-\frac{\mathrm{n}+1}{2} \cdot \mathrm{i}+\frac{(\mathrm{n}+1)(\mathrm{n}+2)}{2 \cdot 3} \cdot \mathrm{i}^{2}-\ldots\right] \text {, }
$$

desprezando $\frac{(\mathbf{n}+\mathbf{1})(\mathrm{n}+2)}{2 \cdot 3} \cdot \mathrm{i}^{2}$ e os sucessivos, temos a seguinte desigualdade:

$$
\begin{aligned}
& \frac{1}{n}>\frac{C_{1}}{C}\left[1-\frac{n+1}{2} \cdot i\right] \\
& \frac{1}{n}>\frac{C_{1}}{C}-\frac{(n+1)}{2} \cdot i \frac{C_{1}}{C} \\
& \frac{(n+1) \cdot i}{2} \frac{C_{1}}{C}>\frac{C_{1}}{C}-\frac{1}{n}\left(x \frac{2 C}{(n+1) C_{1}}\right)
\end{aligned}
$$




$$
\begin{aligned}
& \text { i }>\frac{2}{n+1}-\frac{2 C}{n(n+1) C_{1}} \\
& \text { i }>\frac{2}{n+1}\left[1-\frac{C}{n C_{1}}\right]
\end{aligned}
$$

Podemos, então, tomar como primeira aproximação de $\mathrm{i}$ o valor do segundo membro da desigualdade, isto é:

$$
\mathrm{i}_{\mathrm{o}}=\frac{2}{\mathrm{n}+1}\left[1-\frac{\mathrm{C}}{\mathrm{nC}_{1}}\right]
$$

Em nosso exemplo anterior, teríamos:

$$
i_{o}=\frac{2}{12+1}\left(1-\frac{9.105,46}{12 \times 1.000,00}\right)=0,0371 \text { ou } 3,71 \%
$$

III. A financeira ao vender o crédito ao consumidor, ou seja, o financiamento ao mercado consumidor, diz que a taxa de juros é obtida através da seguinte fórmula:

$$
\begin{aligned}
\mathrm{i}=\frac{\mathrm{N} \times \mathrm{C}_{1}-\mathrm{C}}{\mathrm{C}}, \text { onde } \mathrm{N} & =\text { número de prestações, } \\
\mathrm{i} & =\text { taxa de juros do empréstimo, } \\
\mathrm{C}_{1} & =\text { valor de cada prestação, } \\
\mathrm{C} & =\text { líquido recebido. }
\end{aligned}
$$

Portanto, para $\mathrm{C}=9.105,46, \mathrm{~N}=12$ e $\mathrm{C}_{1}=1.000,00$, teremos:

$\mathrm{i}=\frac{12 \times 1.000,00-9.105,46}{9.105,46}=0,31789$ e a taxa mensal será:

$i_{m}=\frac{0,31789}{12}=0,0265$ ou $2,65 \%$ ao mês.

CRITICA

Observamos aqui que não há para o cálculo da taxa a aplicação fundamental do princípio da amortização de um empréstimo ou seja:

"O montante do capital emprestado deverá ser igual ao montante das prestações." 


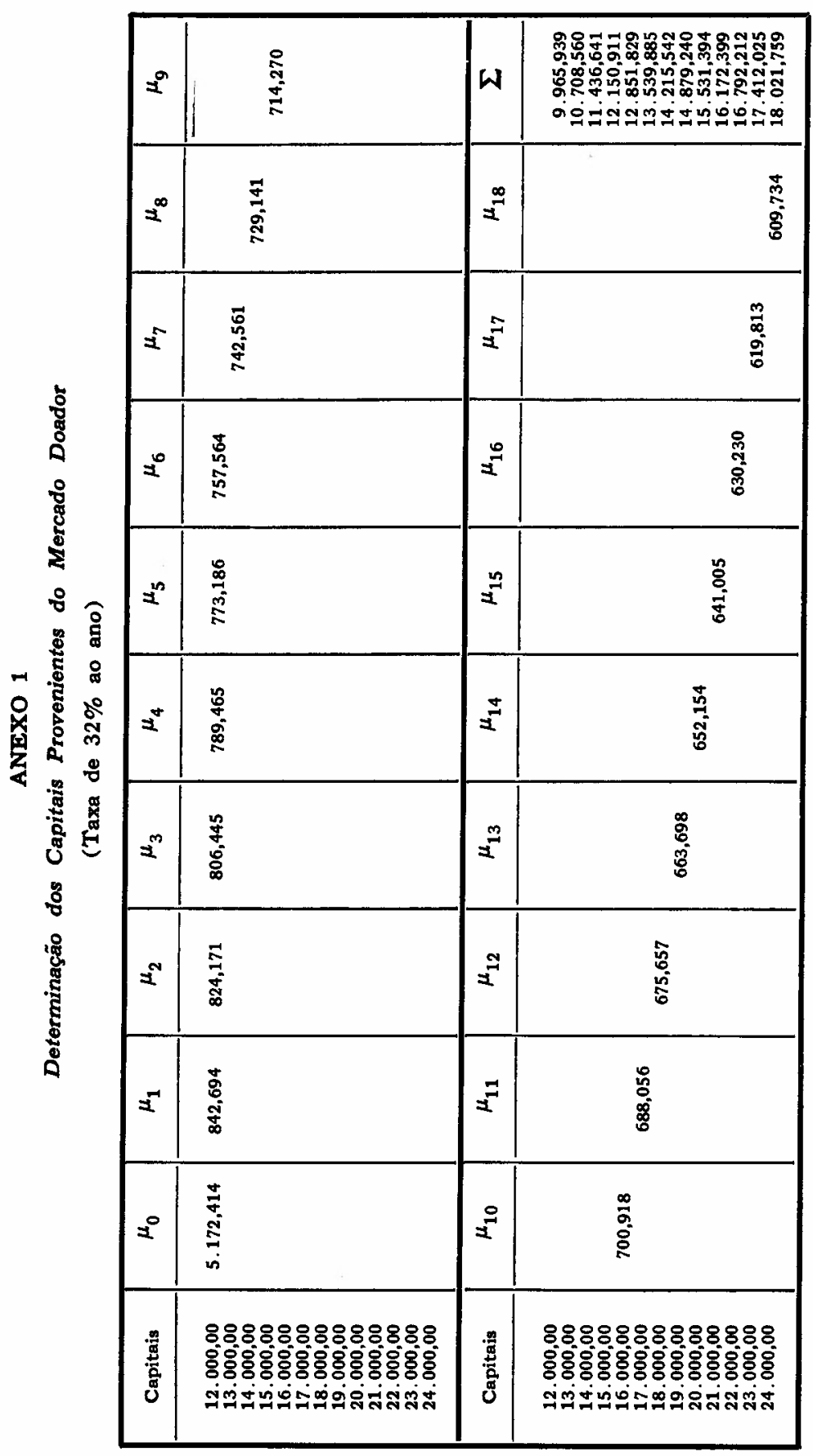




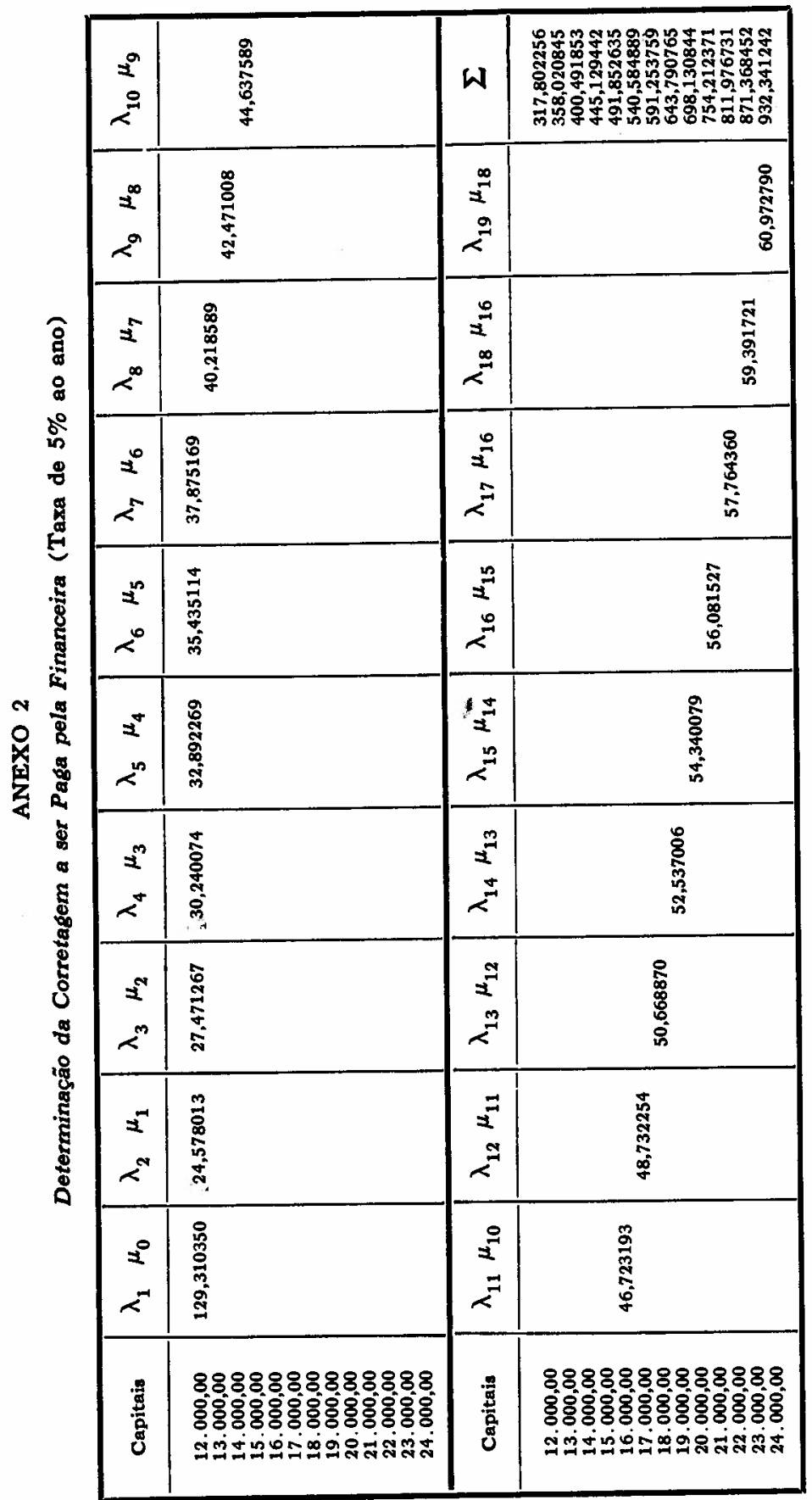




\begin{tabular}{|c|c|c|c|}
\hline $\begin{array}{l}z^{a} \\
z^{2}\end{array}$ & 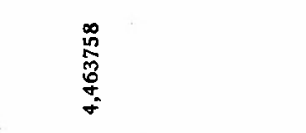 & $\omega$ & 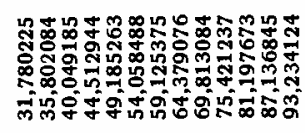 \\
\hline $\begin{array}{l}\sum^{\infty} \\
\sum^{\infty}\end{array}$ & 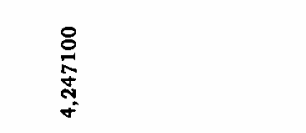 & $\mid \begin{array}{l}\infty \\
\Sigma \\
2 \\
\Sigma \\
\Sigma\end{array}$ & : \\
\hline$\sum_{\Sigma}^{\infty}$ & 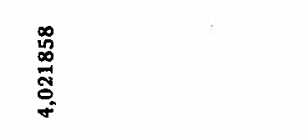 & $\begin{array}{l}= \\
z \\
5 \\
5\end{array}$ & $\begin{array}{l}\mathbb{5} \\
\text { a } \\
\text { o. } \\
\text { in }\end{array}$ \\
\hline $\begin{array}{l}\sum^{0} \\
\Sigma\end{array}$ & 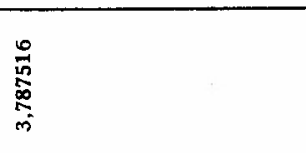 & $\begin{array}{l}0 \\
\Sigma \\
\Sigma \\
\Sigma\end{array}$ & 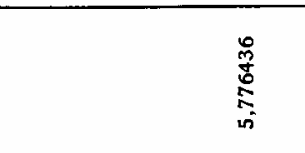 \\
\hline $\begin{array}{l}z^{n} \\
z^{\infty}\end{array}$ & 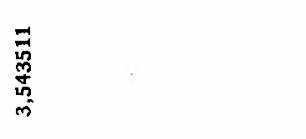 & $\mid \begin{array}{l}2^{n} \\
2 \\
5 \\
2^{2}\end{array}$ & 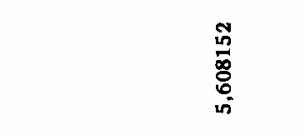 \\
\hline$\sum^{+}$ & 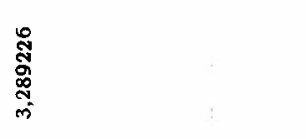 & $\begin{array}{l} \pm \\
\Sigma \\
5 \\
\Sigma\end{array}$ & 㝘 \\
\hline $\begin{array}{l}\sum^{m} \\
a^{+}\end{array}$ & 㝘 & $\begin{array}{l}m \\
z \\
z \\
z\end{array}$ & 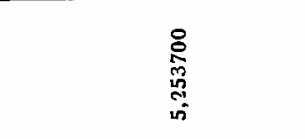 \\
\hline $\begin{array}{l}\sum^{n} \\
\Sigma^{\infty}\end{array}$ & 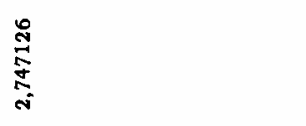 & $\begin{array}{l}z \\
z \\
m \\
z\end{array}$ & 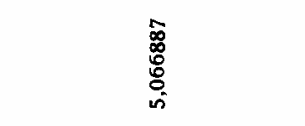 \\
\hline $\begin{array}{l}\Sigma^{-1} \\
\Sigma^{N}\end{array}$ & 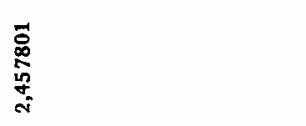 & $\begin{array}{l}z= \\
z \\
z\end{array}$ & 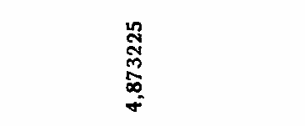 \\
\hline $\begin{array}{l}i^{0} \\
\Sigma\end{array}$ & 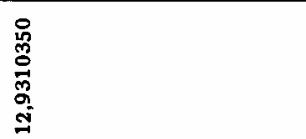 & $\begin{array}{l}= \\
z \\
z\end{array}$ & 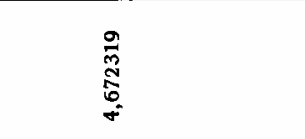 \\
\hline 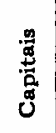 & 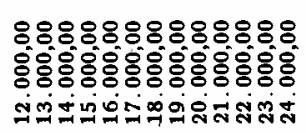 & 总 & 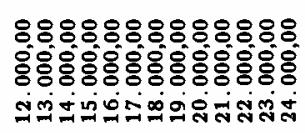 \\
\hline
\end{tabular}




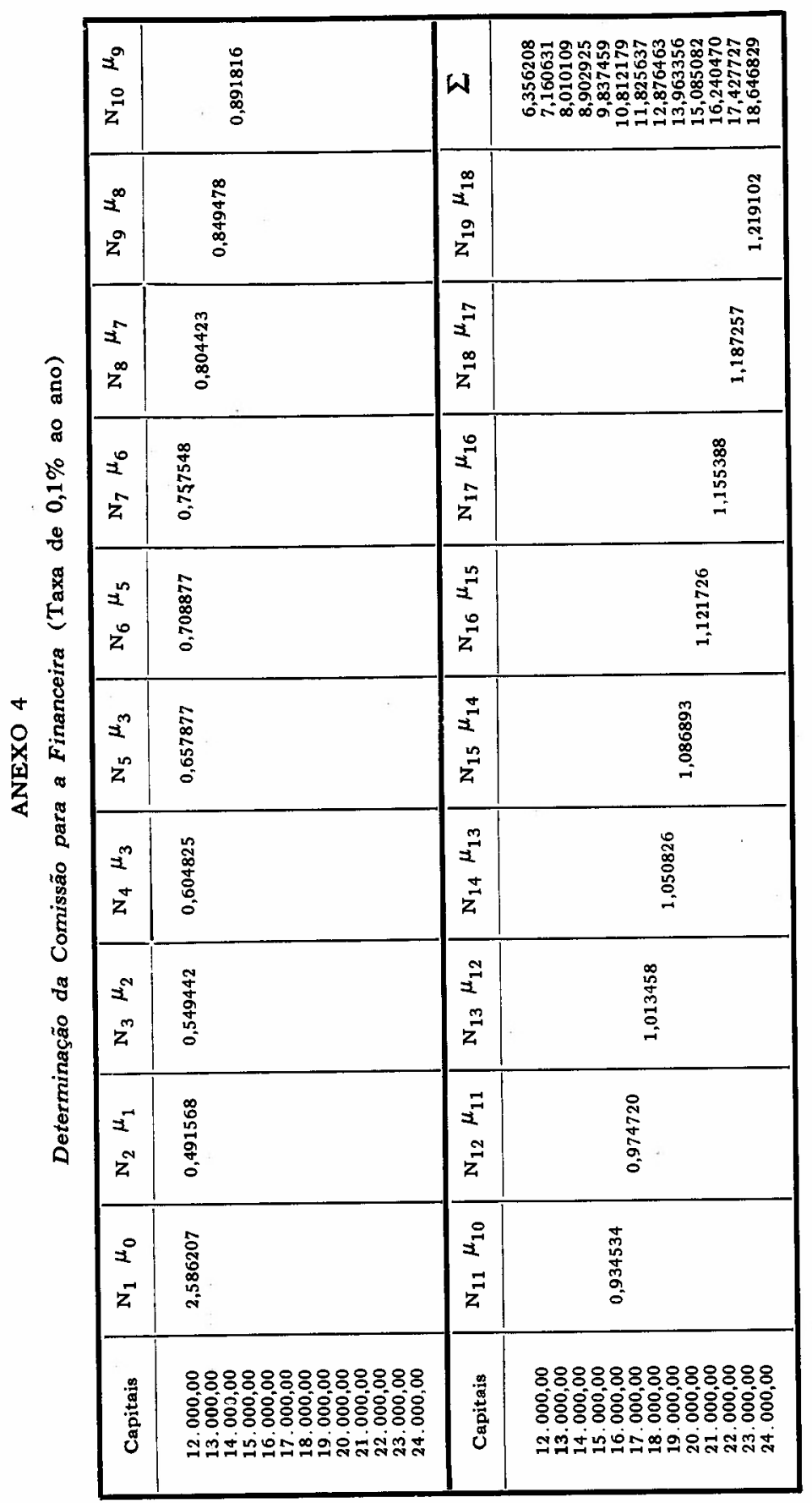



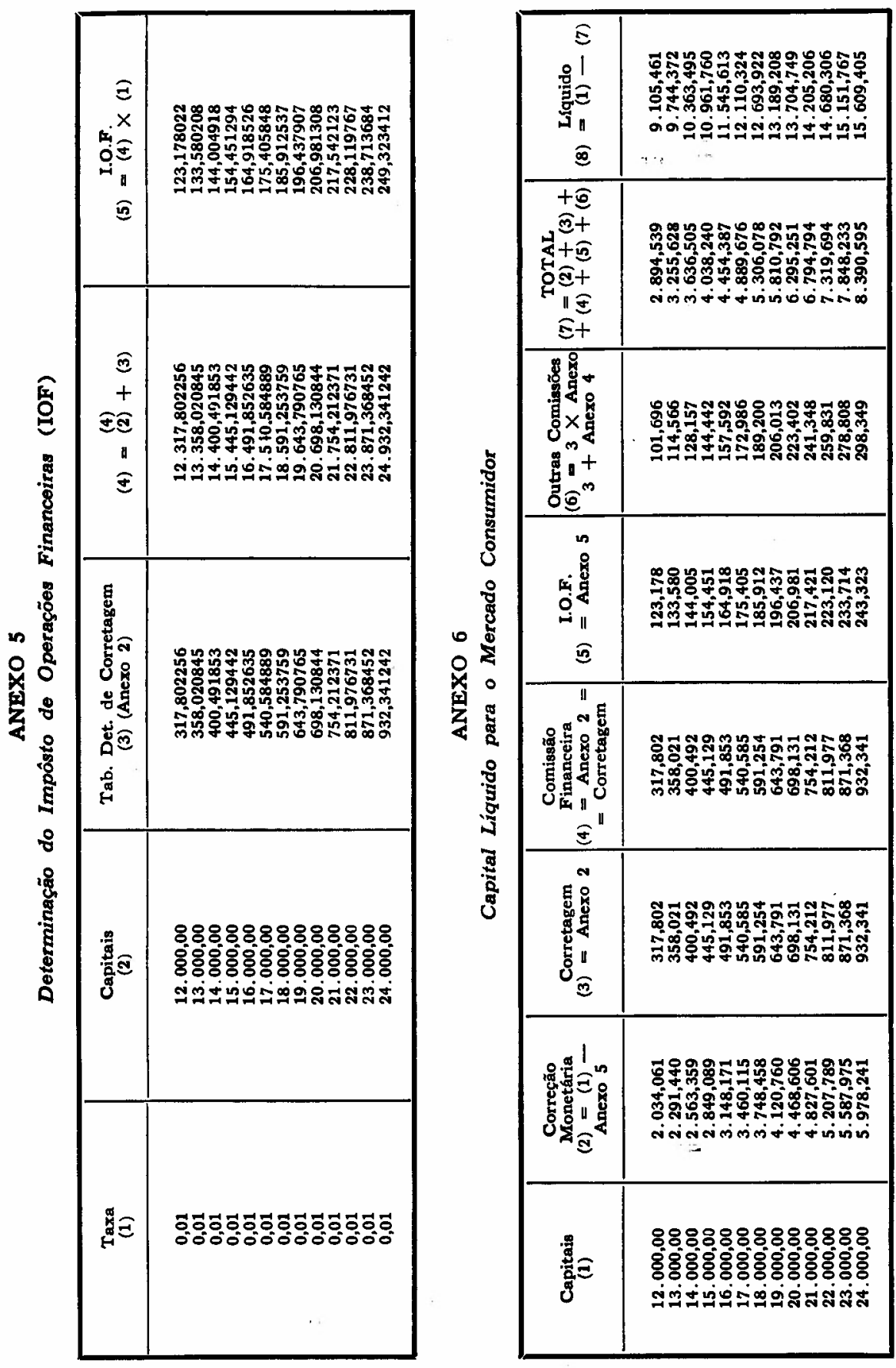